\title{
MANEJO DE MEDICAMENTOS EN CASA, EN PERSONAS CON ENFERMEDAD CRÓNICA NO TRANSMISIBLE (ECNT) Y CUIDADORES
}

\section{HOME CARE MEDICATION IN PEOPLE WITH NONTRANSFERABLE CHRONIC DISEASE (NTCD) AND CAREGIVERS}

\author{
Rosa Coral Ibarra ${ }^{1}$, Jeimy Colmenares Caro ${ }^{2}$, Carmen Lucía Niño ${ }^{3}$
}

\begin{abstract}
${ }^{1}$ Enfermera, Mg. Educación. Facultad de Enfermería, Profesora instructora, Universidad de Ciencias Aplicadas y Ambientales U.D.C.A, Bogotá, Colombia, e-mail: rcoral@udca.edu.co; ${ }^{2}$ Enfermera, Universidad de Ciencias Aplicadas y Ambientales U.D.C.A, Bogotá, Colombia, e-mail: jeimycolmenares@hotmail.com; ${ }^{3}$ Enfermera, Magister en Bioética, Docente Facultad de Enfermería. Universidad de Ciencias Aplicadas y Ambientales U.D.C.A, e-mail: cnino@udca.edu.co
\end{abstract}

Rev. U.D.C.A Act. \& Div. Cient. 18(1): 21-28, Enero-Junio, 2015

\section{RESUMEN}

La enfermedad crónica no trasmisible (ECNT) es la principal causa de morbilidad, a nivel mundial y es un problema de salud pública, que amenaza el desarrollo económico y social de los países. El manejo de la ECNT requiere un régimen terapéutico y farmacológico eficaz para su control. La administración segura de medicamentos en casa es fundamental para prevenir el deterioro de la calidad de vida de las personas y los reingresos hospitalarios, que aumentan los costos para las instituciones y las familias. El objetivo del estudio fue evaluar el manejo de medicamentos en casa, en un grupo de personas con ECNT y en cuidadores. Se empleó un diseño descriptivo transversal. La recolección de la información, se realizó a través de un instrumento de caracterización sociodemográfica y una guía de observación semiestructurada, previamente evaluados por expertos; se aplicó una prueba piloto. El estudio, se practicó con una muestra de 35 personas, quienes cumplieron con los criterios de inclusión, a partir de una población de 71 individuos, que asisten a un Programa de apoyo a la persona mayor, en una Localidad de Bogotá. Se efectuó un análisis descriptivo. La edad de las personas con ECNT fue de 71 a 90 años y la de los cuidadores, de 56 a 70 años. En su mayoría, los dos grupos pertenecían al género femenino, con bajo nivel de escolaridad y en estrato socioeconómico II. Los medicamentos más utilizados son antihipertensivos; las personas almacenan medicamentos vencidos y tienen dificultad para leer la etiqueta.

\section{SUMMARY}

Nontransferable chronic diseases (NTCD) are the leading cause of morbidity worldwide; they are considered a public health problem that threatens economic and social development of many countries. Management of NTCD requires an effective therapeutic and drug regimen to control it. Safe administration of medications at home constitutes the base to prevent life quality weakening of people and hospital readmissions, both increasing costs for institutions and families. The aim of the study was to evaluate medication management at home in a group of people with NTCD and in caregivers. The study used-design was cross sectional descriptive. Data collection was performed using a sociodemographic instrument and semi-structured observation guide previously evaluated by experts and pilot test was applied. Sample for the study corresponded to 35 people, who met the inclusion criteria, from a preliminary 71 people characterized population who attended a Program to support the older person in a district of Bogotá. Data analysis was descriptive. Age of people with NTCD was 71 - 90 years, and caregivers 56 - 70 years. Women were prevalent in two groups, with low levels of education and socioeconomic level II. More used drugs were antihypertensive; people used to stored expired medicines and have difficulty for reading the label.

Key words: Drugs, health, senior, caregivers.

Palabras clave: Medicamentos, hogar, adultos mayores, cuidadores. 


\section{INTRODUCCIÓN}

Según la Organización Mundial de la Salud (OPS, 2012a,b,c), las enfermedades crónicas, como infarto, cáncer, diabetes, hipertensión y Enfermedad Pulmonar Obstructiva Crónica EPOC, representan el $63 \%$ de mortalidad en el mundo. En el 2008, 36 millones de personas murieron de una enfermedad crónica y la mitad fueron mujeres.

En el 2005, 35 millones de personas en el mundo fallecieron a causa de enfermedades crónicas; esta cifra duplica el número de muertes de todos los trastornos infecciosos, incluida la infección por VIH, sida, malaria y tuberculosis. Se prevé que las muertes, debidas a las enfermedades crónicas, aumentarán en un 17\%, para el 2015 (Almanza et al. 2011).

En Colombia, las ECNT son las principales causas de enfermedad y de muerte, sobrepasando, incluso, las causadas por violencia y por accidentes (Almanza et al. 2011). El total de fallecimientos registrados en Colombia, durante 1990 y 2005, por ECNT, pasó de 59\% a 62,6\% (DANE, 2010-2014).

La EPOC es una de las principales causas de mortalidad. En el 2004, se presentaron 64 millones de casos y, en 2005, murieron más de 3 millones de personas, lo cual, representa un $5 \%$ de todas las muertes registradas, para ese año (OMS, 2012a). La diabetes ocupa una de las primeras causas de deceso y se prevé que entre el 2005 y 2030, se multiplicaran (OMS, 2012c).

La hipertensión arterial es otra de las causas de muerte en el mundo, que afecta, tanto a hombres como a mujeres; uno de cada tres adultos sufre de hipertensión y desconocen su enfermedad y uno de cada tres adultos, en tratamiento, no logra mantener su presión arterial baja. Los diagnósticos más frecuentes en los adultos mayores son: hipertensión arterial (1.887) y enfermedad pulmonar obstructiva crónica (653), de un total de 2.540 personas atendidas (Hospital de Suba, Diagnóstico Local de Suba, 2012).

El manejo de medicamentos, en especial, para los adultos mayores, se constituye en un problema, porque existen diversos factores, como déficit de conocimientos, falta de cumplimiento terapéutico, presencia de condiciones mórbidas crónicas, deterioro cognitivo o físico y baja escolaridad, que conllevan, con mayor frecuencia, a efectos adversos (Rojas Mora \& Sáenz Campo, 2000).

Se ha reportado que los adultos mayores suelen guardar grandes cantidades de medicamentos. Se encontró una media de ocho medicamentos por hogar y hasta el 30\% de los pacientes tenían, por lo menos, diez medicamentos (Abou-A, 2003). El almacenamiento de grandes cantidades de medicamentos los pone en riesgo de cometer errores, como envenenamiento accidental y reacciones adversas (Kheir et al. 2011). Además, los adultos mayores, se han constituido en grandes consumidores de medicamentos, por las pluri-patologías que padecen, se auto medican y están expuestos a reingresos hospitalarios y mayor consumo de servicios de salud (Arriagada et al. 2008).

Se calcula que más de la mitad de los medicamentos se prescriben, se dispensan o se venden de forma inapropiada y la mitad de los pacientes no los toman correctamente (OMS, 2010). Los pacientes deben adquirir experiencia en el manejo de medicamentos, con ejercicios prácticos, que les permitan observar, corregir y verificar las habilidades en la administración y el equipo de salud debe evaluar, en forma continua, esta actividad. Se reconoce el rol del profesional de enfermería en la educación de los cuidados en el hogar (Souzai \& Zanettill, 2009).

Teixeira de Souza et al. (2001) refieren que es importante que los profesionales de salud participen en la educación para el autocuidado de pacientes con diabetes en casa, con el fin de reducir la sobrecarga de las complicaciones crónicas.

El objetivo del estudio fue evaluar el manejo de medicamentos en casa, en un grupo de personas con ECNT y en cuidadores.

\section{MATERIALES Y MÉTODOS}

Estudio descriptivo transversal. La recolección de la información, se realizó a través de una guía de observación y una encuesta sociodemográfica, evaluada por expertos y con la aplicación de una prueba piloto. La población: corresponde a 71 personas, con ECNT y cuidadores, quienes fueron caracterizados en el 2012. La muestra corresponde a 19 cuidadores y 16 personas con ECNT. El muestreo fue intencional de acuerdo a los siguientes criterios de inclusión:

Personas con ECNT: Mayores de 65 años, con diagnóstico de hipertensión, diabetes o enfermedad pulmonar obstructiva crónica, que manejaban uno o más medicamentos en casa y tener un cuidador vinculado a la institución (Secretaría de Integración Social de una Localidad de Bogotá, D.C.), en la cual, se maneja la política de atención al adulto mayor y al anciano.

Cuidadores de personas con ECNT: Vinculados a la institución, mayores de 18 años y manejar uno o más medicamentos en casa.

Consideraciones éticas: El estudio fue aprobado por el Comité de Ética de la Universidad y contó con la aprobación de las directivas de la Secretaría de Integración Social. Se solicitó el consentimiento informado al grupo de estudio, la participación fue voluntaria y se mantuvo, en todo momento, 
la privacidad y la confidencialidad. Se aplicaron los principios ético- legales de los procesos de investigación. Se tuvo en cuenta la Resolución 8340 del Ministerio de Salud y los principios de la Declaración de Helsinki y la CIOMS, para la investigación con personas.

\section{RESULTADOS Y DISCUSIÓN}

Para las personas que participaron en el estudio con ECNT, el rango de edad fue entre 71 y 90 años y la edad de los cuidadores, entre 40 y 70 años.

El $87,5 \%$ de las personas con ECNT y el $100 \%$ de las cuidadoras eran mujeres, lo cual, coincide con el Diagnóstico de la Localidad (Diagnóstico Local de Suba, 2012).

Según la OMS (2012b), la depresión es un trastorno mental frecuente, que afecta a más de 350 millones de personas en el mundo y, en mayor grado, a la mujer; esta condición para García de Alba et al. (2011 hace que los cuidadores de personas con ECNT presenten altos niveles de depresión y de ansiedad, lo que varía en función de la duración e intensidad de la situación clínica de la persona a su cuidado.

El presente estudio mostró que el $43,75 \%$ de las personas con ECNT son analfabetas y el 36,84\% de los cuidadores han cursado, al menos, un grado de bachillerato. Los adultos mayores que no aprobaron ningún año de educación formal fue mínimo (0,1\%); el $28 \%$ aprobó algún año de secundaria y, el 23\%, algún año de educación superior (Alcaldía Mayor de Bogotá, 2013). Los datos de la Localidad de los sujetos de estudio muestran que $4.554(1,2 \%)$ hombres y 3.935 $(0,9 \%)$ mujeres son analfabetas (Hospital de Suba, 2012); un estudio mostró que el $74,4 \%$ no sabían leer (Gautério \& Costa, 2012). Otra investigación reveló que los adultos mayores no tenían ningún grado de escolaridad (Manrique \& Fernández, 2011). De 300 adultos mayores, el 15\% no tenían ningún grado de escolaridad y $50 \%$, primaria incompleta (Zavala et al. 2006).

En su mayoría, las personas que participaron en el estudio pertenecen al estrato dos. Según el informe de la Localidad, 80.597 hogares tienen necesidades básicas insatisfechas; 19.879 corresponden a hogares con personas de más de 60 años de edad (Alcaldía Mayor de Bogotá, D.C. 2010). al respecto, Manrique \& Fernández (2011) encontraron que el 53,3\% de los adultos mayores pertenecen al estrato uno. Lo anterior implica que las condiciones socio-económicas deficientes pueden afectar, de alguna manera, el estado de salud de las personas y, por ende, sus prácticas de autocuidado.

Los participantes del estudio utilizan antihipertensivos, analgésicos, laxantes, antiparasitarios, hidantoinas y vitaminas.
Al respecto, se ha estimado que las personas que sufren de tres o más enfermedades crónicas presentan polifarmacia (Villardón et al. 2011; Gautério \& Costa, 2012; Rojas et al 2008), que afecta la adherencia al tratamiento (Pavón \& González, 2010). Se presenta, con mayor frecuencia, en mujeres (Marín, 2010; Mira \& Navarro, 2012; Campany, 2006). Otros estudios han mostrado que el $88 \%$ de las hospitalizaciones en adultos mayores, se dan por interacción medicamentosa (Peralta \& Medina, 2013).

De acuerdo a los resultados obtenidos, el $93,75 \%$ de las personas guardan los medicamentos en la habitación. Un estudio mostró que los adultos conservan los medicamentos en las habitaciones, seguido del refrigerador y la cocina (Kheir et al. 2011). Vargas \& Sáenz (2013) no evidenciaron diferencias significativas, si las personas los guardan en el dormitorio, seguido de la cocina y el baño.

Además, se estableció que el $88 \%$ de las personas preservan los medicamentos en bolsas plásticas. Al respecto, Kheir et al. (2011) encontraron que la mayoría de las personas no tienen un lugar específico para guardarlos; un $45,5 \%$ de los pacientes acumulan los medicamentos y un $34 \%$ guardan los que no están prescritos (Mora et al. 1999).

En relación a la conservación de los medicamentos, se sugiere que se guarden en lugares frescos, secos y sin cambios de temperatura; lo ideal es almacenarlos entre 8 y $15^{\circ} \mathrm{C}$ y según recomendaciones en la etiqueta (Casamitjana, 2012; Campany, 2006; Correa, 2001).

De acuerdo a lo anterior, es importante que el equipo de salud y, en especial, el profesional de enfermería que realiza su labor en el ámbito comunitario, realice seguimiento a las personas con ECNT, que incluya la administración y la disposición final de los medicamentos.

El estudio arrojó que el $81,25 \%$ de las personas con ECNT y el $84,21 \%$ de los cuidadores, no utilizan ninguna forma de organizar los medicamentos; sin embargo, un mínimo porcentaje los guarda por horario, lo que implica el riesgo del manejo inadecuado del régimen terapéutico. Vargas \& Sáenz (2013) apreciaron que los adultos mayores se toman los medicamentos sin horario establecido; los errores también están relacionados con la falta de conocimiento y el olvido en la toma de los mismos. Es frecuente en adultos mayores, porque no tienen en cuenta los horarios o las prescripciones médicas.

La tabla 1 muestra que la mayoría de las personas con ECNT, $62,50 \%$, tenía la fórmula médica, de los cuales, el $50 \%$ estaba vigente. En el grupo de cuidadores, el 73,68\% presentó la fórmula médica de la persona que cuidaban y, de este total, el 63,15\% la tenía vigente; los adultos mayores 
suelen auto administrarse los medicamentos (Rojas-Mora \& Sáenz-Campos, 2000). En otros casos, tienen fórmulas de medicamentos que no utilizan (Thompson \& Stewart, 2001) o, por el contrario, consumen medicamentos que no están formulados. Esta información muestra la necesidad de hacer seguimiento, en especial, a quienes se auto medican y utilizan varios medicamentos, para el control de la enfermedad. El control médico, se debe realizar cada 3 a 6 meses, según el riesgo (Oliveira et al. 2012).

Cabe anotar que el $50 \%$ de las personas del estudio y el $57,89 \%$ de los cuidadores guardan medicamentos vencidos en casa. En esta investigación, se detectó a una persona que guardaba y utilizaba grandes cantidades de medicamentos vencidos.
Al respecto, un estudio realizado en cinco regiones de Arabia Saudita y otros países del Golfo, mostró que de un total 1.641 familias, el $25,8 \%$ y el $41,3 \%$, respectivamente, tenían medicamentos deteriorados o vencidos en el hogar y acumulan más de tres cajas de la misma medicación (Rojas et al. 2008); los adultos mayores de 65 años cometen errores en la toma de medicamentos. 1,5 millones de eventos adversos prevenibles, se producen cada año en EE.UU, resultando en 7.000 muertes (Center for Home Care Policy And Research, 2002), lo que implica un riesgo potencial de toxicidad, que pone en peligro la vida de los pacientes (Correa, 2001).

El presente estudio mostró que las personas con ECNT no utilizan ninguna medida de seguridad para almacenar

Tabla 1. Orden médica y fecha de la última prescripción.

\begin{tabular}{|l|l|l|l|l|}
\hline ORDEN MÉDICA & PACIENTE & $\%$ & CUIDADOR & $\%$ \\
\hline $\mathrm{Si}$ & 10 & $62,50 \%$ & 14 & $73,68 \%$ \\
\hline No & 6 & $37,50 \%$ & 5 & $26,32 \%$ \\
\hline TOTAL & 16 & $100 \%$ & 19 & $100 \%$ \\
\hline FECHA ÚLTIMA PRESCRIPCIÓN & PACIENTE & $\%$ & CUIDADOR & $\%$ \\
\hline Últimos tres meses & 8 & 50 & 12 & $63,15 \%$ \\
\hline Últimos seis meses & 1 & $6,25 \%$ & 1 & $5,26 \%$ \\
\hline Más de seis meses & 1 & $6,25 \%$ & 1 & $5,26 \%$ \\
\hline No tienen orden medica & 6 & $37,50 \%$ & 5 & $26,32 \%$ \\
\hline TOTAL & 16 & $62,50 \%$ & 19 & $73,68 \%$ \\
\hline
\end{tabular}

los medicamentos. De los cuidadores, solamente el 5,26\% utilizaba alguna medida. Kheir et al. (2011) evidenciaron que los medicamentos almacenados en el hogar los consumen otras personas, diferentes a la que recibió la prescripción.

En el informe de la Línea de Acción de Medicamentos del 2011, de la Secretaría Distrital de Salud de Bogotá (2011), se notifica que en el 2010, se presentaron 1.672 casos de intoxicaciones, de los cuales, el $79 \%$ fue por el principio activo y el $21 \%$, por mezclas.

Se estableció, que el 31,25\% de las personas con ECNT tienen mucha dificultad para la lectura correcta de la etiqueta del medicamento y el 18,75\%, no pueden leerla. En relación a los cuidadores, el 47,37\% tienen mucha dificultad; sin embargo, con lentes, es menor.

Según la Organización Panamericana de la Salud, los adultos mayores presentan problemas de la visión con la edad, como resultado del deterioro funcional, lo que afecta la calidad de vida (OPS, 2013). El 85\% de los adultos mayores que consumen medicamentos, de forma habitual, tienen riesgo de cometer errores. Se ha encontrado que un $57 \%$ de los productos disponibles en casa no tienen instrucciones para su empleo (Rojas-Mora \& Sáenz-Campos, 2000). Una investigación sustenta que los errores aumentan, proporcionalmente, con el número de medicamentos, que se tengan que consumir (Center for Home Care Policy and Research, 2002). Las personas que no leen las etiquetas es porque no entienden la información, porque el color es poco atractivo o la letra es muy pequeña.

En el presente estudio, se identificó que el $43,75 \%$ de las personas con ENCT utilizaban algunos mecanismos para diferenciar los medicamentos, cuando no podían leer, no sabían leer o no podían ver, entre ellos: carteleras con los nombres de los medicamentos y horarios, utilización de cinta de enmascarar alrededor de la caja, dejar listos los medicamentos del día en un plato o la identificación por colores. La cronicidad de la enfermedad, en personas con retinopatía diabética, no permite ver los colores, por lo tanto, presentan dificultad para diferenciar las etiquetas. 
La tabla 2 evidencia que el $50 \%$ de las personas con ECNT no tienen dificultad para retirar la envoltura del medicamento; sin embargo, hubo personas que tenían dificultad. Cabe mencionar que las mujeres tienen menos impedimento, en relación con los hombres (Rojas-Mora \& Sáenz-Campo, 2000).
Un estudio también evidenció, que de 40 adultos mayores, el $13 \%$ de mujeres y el $12 \%$ de hombres incumplían el régimen terapéutico (Rojas et al. 2000), lo que permite considerar que muchas hospitalizaciones se deben al consumo de dosis menores a las prescritas (Peralta \& Medina, 2013) o duplicidad (Center for Home Care Policy and Research, 2002).

Tabla 2. Dificultad de las personas con ECNT y cuidadores para retirar los medicamentos de la envoltura o recipientes.

\begin{tabular}{|l|l|l|l|l|}
\hline $\begin{array}{l}\text { DIFICULTAD AL RETIRAR EL MEDICAMENTO DE } \\
\text { LA ENVOLTURA }\end{array}$ & PACIENTE & $\%$ & CUIDADOR & $\%$ \\
\hline Ninguna & 3 & $18,75 \%$ & 15 & $78,95 \%$ \\
\hline Poca dificultad & 8 & $50 \%$ & 4 & $21,05 \%$ \\
\hline Mucha dificultad & 5 & $31,25 \%$ & 0 & $0 \%$ \\
\hline TOTAL & 16 & $100 \%$ & 19 & $100 \%$ \\
\hline $\begin{array}{l}\text { DIFICULTAD AL RETIRAR EL MEDICAMENTO } \\
\text { DEL RECIPIENTE }\end{array}$ & PACIENTE & $\%$ & CUIDADOR & $\%$ \\
\hline Ninguna & 2 & $12,50 \%$ & 5 & $26,32 \%$ \\
\hline Poca dificultad & 0 & $0 \%$ & 1 & $5,26 \%$ \\
\hline Mucha dificultad & 1 & $6,25 \%$ & 0 & $0 \%$ \\
\hline No aplica & 13 & $81,25 \%$ & 13 & $68,42 \%$ \\
\hline TOTAL & 16 & $100 \%$ & 19 & $100 \%$ \\
\hline
\end{tabular}

La técnica utilizada para la administración de inhaladores por parte de los las personas y cuidadores del estudio es correcta; sin embargo, el tiempo de espera entre cada inhalación es diferente (5, 10, 20 y 30 segundos); Aranga et al. (1999) identificaron que un $37,5 \%$, administra la siguiente dosis sin tiempo de espera y Robinson (1980) reveló que las mujeres adultas mayores cometen más errores que los hombres. Las personas con diagnóstico de EPOC, no tienen una técnica adecuada para utilizar los inhaladores; se ha encontrado que hasta el $76 \%$ de los pacientes, que utilizan inhalador de dosis medida, cometen, al menos, un error y, de éstos, un $28 \%$ a $30 \%$, incurren en descuidos, que comprometen la eficacia del tratamiento (Molimard et al. 2003). En una evaluación a un grupo de pacientes con EPOC y asma, se registró que los pacientes se equivocaban, al menos una vez, en la técnica de inhalación (Souza \& Zanettill, 2009).

Con relación al uso de otros medicamentos, en el presente estudio, se halló que solo un cuidador aplicaba insulina y, en general, cumplía con la técnica, a excepción de la rotación del sitio de administración. Se ha reportado la presencia de complicaciones subcutáneas en la administración de la insulina, que están asociadas con la técnica inadecuada (Souza \& Zanettill, 2009), lo cual, puede alterar los efectos terapéuticos esperados (Campany, 2006). Se encontró que hasta el $88 \%$ de los pacientes cometen errores en la administración de la insulina, asociados a olvidos, confusiones o equívocos durante la administración de la insulina u otros medicamentos (Mira \& Navarro, 2012).

Las enfermeras deben estar comprometidas con una práctica profesional al lado de personas, familias o comunidades y son responsables de ofrecer cuidados específicos, basados en las necesidades que viven las personas, ante diversas experiencias de salud (Zárate, 2004). La profesión de enfermería, se orienta a la práctica de la enfermera sobre lo que dirige su juicio clínico y sus decisiones profesionales. Es importante la implementación de estrategias, que faciliten la organización e identificación de los medicamentos, para minimizar y prevenir complicaciones derivadas de los efectos adversos, la automedicación y el inadecuado control del tratamiento. 
Es imperativo que se realice capacitación a la persona con ECNT, así como a los cuidadores y a la red de apoyo, de manera integral y que contribuya a la disminución de la carga de la enfermedad. En Colombia, se ha venido trabajando para desarrollar un modelo de atención dirigido a diferentes actores que participan en el cuidado: instituciones, profesionales, personas con ECNT y cuidadores.

Reconocimiento: Los resultados de este trabajo contribuyen al "Programa para la disminución de la carga de la enfermedad crónica no transmisible en Colombia", aprobado por COLCIENCIAS, en la Convocatoria 537 de 2011, liderado por la Universidad Nacional de Colombia, en conjunto con la Universidad de Santander UDES, Universidad Mariana de Pasto y Universidad de Ciencias Aplicadas y Ambientales U.D.C.A. Conflicto de intereses: El manuscrito fue preparado y revisado con la participación de todos los autores, quienes declaramos que no existe ningún conflicto de intereses, que ponga en riesgo la validez de los escritos presentados.

\section{BIBLIOGRAFÍA}

1. ABOU-A, S.A. 2003. Una evaluación económica de la extensión de la utilización de los medicamentos y desperdicio entre las familias en Arabia Saudita y los países del Golfo Pérsico. Pudmed. Disponible desde Internet en: http://www.ncbi.nlm.nih.gov/ pubmed/12809973 (con acceso 25/11/2013).

2. ALCALDÍA MAYOR DE BOGOTÁ. 2013. Atención Integral para Personas Mayores: Disminuyendo la Discriminación y la Segregación Socioeconómica. P.1-34. Disponible desde Internet en: http://www. integracionsocial.gov.co/anexos/documentos/ proyectosbogotahumana Atención Integral para personas mayores disminuyendo la discriminación segregacion.pdf (con acceso 10/12/2013).

3. ALCALDÍA MAYOR DE BOGOTÁ, 2010. Política pública social para el envejecimiento y la vejez en el Distrito Capital 2010-2025. 158p.

4. ALMANZA, R.; MONTES, F.; LOPERA, V.; GRAJALES, I.; HOYOS, E.; SALAS, C.; VALLEJO, I.; ARRUBLA, M.; RESTREPO, C.; GUZMAN, L. 2011. Análisis de la situación de salud de Medellín 2010. Rev. Salud Pública Medellín. (Colombia). 26(2):61-74.

5. ARANGA, A.; LÓPEZ, S.; RAMOS, J.; GARCÍA, G.; CLOPES, A.; BONAL, J. 1999. Valoración de los conocimientos y utilización de inhaladores en pacientes hospitalizados. Rev. Farmacia Hospitalaria. (España). 23(5):307-312.
6. ARRIAGADA, L.; JIRÓN, M.; RUÍZ, I. 2008. Uso de medicamentos en el adulto mayor. Rev. Hosp. Clín. Univ. (Chile). 19:309-317.

7. CAMPANY, M. 2006. Conservación y administración de medicamentos. Prevención de problemas relacionados con medicamento. Rev. Ámbito Farmacéutico. (Argentina). 25(8):70-77.

8. CASAMITJANA, N. 2012. Centro de Información del Medicamento. Colegio de Farmacéuticos de Barcelona. (España). Disponible desde Internet en: http://www.farmaceuticonline.com/ es/el-medicamento/610-conservacion-de-losmedicamentos?start $=3$ (con acceso 17/12/2013).

9. CENTER FOR HOME CARE POLICY AND RESEARCH. 2002. Preventing Medication Errors in Home Care. $6 \mathrm{p}$.

10. CORREA, S. 2001. Los Medicamentos Vencidos: ćqué necesitamos saber? Facultad de ciencias química. Disponible desde Internet en: http://cime.fcq.unc. edu.ar/vencimientosII.htm (con acceso 16/12/2013).

11. DEPARTAMENTO NACIONAL DE PLANEACIÓN DANE-. 2010-2014. Plan Nacional de Desarrollo. Disponible desde internet en: WWW.DNP.GOV.CO/ LINKCLICK.ASPX?FILETETICKET =U5I1XXLPFJA\% 3D\&TABID =1238 (con acceso 10/02/2013).

12. GARCÍA DE ALBA, J.; CASTAÑEADA, E.; PANDO, M. 2011. Depresión en asistentes médicas: análisis de los factores de riesgo sociolaborales. Psicología y Salud. 21:73-78.

13. GAUTÉRIO, D.; COSTA, S. 2012. Caracterización de ancianos usuarios de medicación residentes en hogar geriátrico. Rev. Da Escola de Enfermagem da USP. 46(6):1395-1400.

14. HOSPITAL DE SUBA. Diagnóstico Local de Suba. 2012. p.1-222. Disponible desde Internet en: http://www.esesuba.gov.co/joomla/portal/images/ stories/Documentos/Salud_Publica/diagnostico\%20 2011\%20version\%204\%20de\%20abril\%202012.pdf (con acceso 31/07/2013).

15. KHEIR, N.; AWAISU, A.; KIDA, M.; ADAM, A. 2011. Un estudio exploratorio sobre medicamentos en los hogares de Qatar. 36:4 702-702. Disponible dese Internet en: http:/www.ncbi.nlm.nih.gov/ pubmed/22279414 (con acceso 04/08/14). 
16. MARÍN, M. 2010. Los diagnósticos de enfermería s de pacientes de edad avanzada que usan múltiples fármacos. Rev. Esc. Enferm. (Brasil). 44(1):46-51.

17. MANRIQUE, F.; FERNÁNDEZ, R. 2011. Agencia de auto cuidado y factores básicos condicionantes en adultos mayores. Avances Enferm. 29(1):30-41.

18. MIRA, J.; NAVARRO, I. 2012. Frecuencia de errores de los pacientes con su medicación. Rev. Panam. Salud Pública. 31(2):1-14.

19. MORA, L.; ROJAS, L.; SÁENZ, D. 1999. Análisis de la disposición y uso de medicamentos por adultos mayores a nivel domiciliar. Disponible desde Internet en: www.binasss.sa.cr/revistas/farmacos/v12n1/art3. pdf (con acceso 25/11/2013).

20. MOLIMARD, M.; RAHERISON, C.; LIGNOT, S.; DEPONT, F.; ABOUELFATH, A.; MOORE, N. 2003. Assessment of Handling of Inhaler Devices in Real Life: An Observational Study in 3811 Patients in Primary Care. J. Aerosol Medicine. 16(3):249-254.

21. OLIVEIRA ATUNES DE, M.; FRANCISCO STOLSES BERGAMO, P.M.; COSTA SARMENTO, K.; BARROS DE AZEVEDO, M.B. 2012. Automedicação em idosos residentes em Campinas, São Paulo, Brasil: prevalência e fatores associados. Cad. Saúde Pública 28(2):335-345.

22. ORGANIZACIÓN MUINDIAL DE LA SALUD. 2012a. Enfermedad Pulmonar Obstructiva Crónica. (EPOC). Disponible desde Internet en: http://www.who. int/mediacentre/factsheets/fs315/es/ (con acceso 20/02/2013).

23. ORGANIZACIÓN MUINDIAL DE LA SALUD. 2012b. La depresión. Disponible desde Internet en: http:// www.who.int/mediacentre/factsheets/fs369/es/ (con acceso 22/01/2013).

24. ORGANIZACIÓN MUINDIAL DE LA SALUD. 2012c. Diabetes. Disponible desde Internet en: http://www. who.int/mediacentre/factsheets/fs312/es/ (con acceso 20/02/2013).

25. ORGANIZACIÓN MUNDIAL DE LA SALUD. 2010. Medicamentos: uso irracional de los medicamentos. Disponible dese Internet en: http://www.who.int/ mediacentre/factsheets/fs338/es/ (con acceso 01/12/2013).
26. ORGANIZACIÓN PANAMERICANA DE LA SALUD. 2013. Encuesta sobre diabetes, hipertensión y factores de riesgo de las enfermedades: Centroamérica. Disponible desde Internet en: http://www.paho. org/hq/index.php?option $=$ com_content\&view $=$ ar ticle\&id $=3070 \&$ Itemid $=1 \&$ lang $=$ es $\quad($ con acceso 20/02/2013).

27. PAVÓN, J.; GONZÁLEZ, R. 2010. Museo de la Farmacia Habanera y el adulto mayor: cita con el patrimonio farmacéutico y con temas de educación para la salud. Disponible desde Internet en: http://www.bvs. sld.cu/revistas/far/vol_44_4_10/far15410.htm (con acceso 17/12/2013).

28. PERALTA, M.; MEDINA, G. 2013. Guía de práctica clínica prescripción farmacológica en el adulto mayor. Rev. Méd. Inst. Méx. Seguro Soc. 51(2):228-239.

29. ROBINSON, A. 1980. Prácticas de medicamentos recetados de las personas mayores. Pubmed. 3(3):131-136.

30. ROJAS-MORA, L.; SÁENZ-CAMPOS, D. 2000. Análisis de la condición funcional de los adultos mayores para el manejo domestico de los medicamentos. Acta Méd. Costarric. 42(3):115-120.

31. ROJAS G., M.; RUIZ A., I.; CARRASCO M., V.H.; MOLINA Y., J.C. 2008. Caracterización de pacientes y de uso de medicamentos en un grupo de adultos mayores asistentes a una actividad promocional de geriatría. Rev. Hops. Clin. Univ. (Chile). 19:318-323.

32. SECRETARÍA DISTRITAL DE SALUD. 2011. Línea de acción de medicamentos seguros. Disponible desde Internet en: http://saludpublicabogota.org/ wiki/index.php?title $=$ P\%C3\%A1gina_principal (con acceso 06/01/2013).

33. SOUZAI, C; ZANETTILL, M. 2009. Insulina: Un enfoque fundamental en la educación de la diabetes. Rev. Latino-am Enfermagem. 22(4):385-391.

34. TEIXEIRA DE SOUZA, C.R; ZANETTILL, M.L.; RIBEIRO PRADO, K. 2001. Reutilización de jeringas desechables: frecuencia y costos para la administración de insulina en el hogar. Rev. LatinoAm. Enfermagem. 9(5):47-54.

35. THOMPSON, H.; STEWART, K. 2001. Prácticas de uso de medicamentos recetados de las personas mayores no institucionalizadas. Disponible desde Internet en: http://www.ncbi.nlm.nih.gov/pubmed /term 
$=$ Prescription + medication + use + practices + amon $\mathrm{g}+$ noninstitutionalised+older + persons (con acceso 26/11/13).

36. VARGAS, C.; SÁENZ, D. 2013 Análisis de utilización de medicamentos en personas adultas mayores. Acta Méd. Costarric. 55(4):1-11.

37. VILLARDÓN, P.; MARTÍN J.; BERNA, E. 2011. Factores asociados a la polifarmacia en población anciana no institucionalizada. Análisis de la sub-muestra de la Encuesta Nacional de Salud 2006 para personas mayores de Castilla y León. (España). Rev. Española Geriatría Gerontología. 46(06):303-306.
38. ZÁRATE, R. 2004. La gestión del cuidado de enfermería. Granada, primavera verano. Índex de Enfermería 4445:42-46.

39. ZAVALA, M.; VIDAL, D.; CASTRO, M.; QUIROGA, P.; KLASSEN, G. 2006. Funcionamiento social del adulto mayor social. Rev. Ciencia y Enfermería. 12(2):53-62.

Recibido: Junio 8 de 2014

Aceptado: Abril 15 de 2015

Cómo citar:

Coral Ibarra, R.; Colmenares Caro, J.; Niño, C.L. 2015. Manejo de medicamentos en casa en personas con enfermedad crónica no transmisible (ECNT) y cuidadores. Rev. U.D.C.A Act. \& Div. Cient. 18(1): 21-28. 